SCIREA Journal of Electrical Engineering

http://www.scirea.org/journal/DEE

November 1, 2021

Volume 6, Issue 4, August 2021

\title{
An experimental research of the ultra-wideband pulse propagation in a transdirectional coupler based on coupled striplines
}

\section{Nickolay Malyutin, Evgeniy Trenkal, Aleksandr Sychev}

Tomsk State University of Control System and Radioelectronics, Tomsk, Russian Federation

Email:ndm@main.tusur.ru,trenkal@mail.ru, ans@main.tusur.ru

\begin{abstract}
The propagation of short picosecond pulses in coupled strip lines with a phase velocity ratio of odd and even modes of 3:1 is considered. The feature of the separation of the input pulse between the three output ports without significant loss of energy due to reflection from the input in transdirectional couplers based on coupled strip lines is experimentally shown. As a result of the analysis, it was found that the spectral components of the pulse are separating between the ports of the transdirectional coupler.
\end{abstract}

Keywords: striplines, unbalanced coupling, transdirectional coupler, pulsed action, pulse propagation, spectral characteristics. 


\section{INTRODUCTION}

The theory of coupled strip lines (CSL) with unbalanced electromagnetic coupling began to develop in the works of the 60s [1, 2]. In [1] (1967), an analysis of the main properties of coupled lines with equal phase velocities in odd and even modes were made. The inequality of the velocities in even $v_{e}$ and odd $v_{o}$ modes depends on the design of the cross section of the coupled lines. The authors of [2] (1969) obtained the matrix parameters of identical coupled strip lines with inequality $v_{e} \neq v_{o}$ and showed on the examples of several schemes a qualitative change in the characteristics of devices on coupled lines with inequality in the electrical lengths of segments of coupled lines for even and odd excitation of conductors.

To determine the degree of phase velocity inequality, the ratio of capacitive and inductive coupling coefficients is introduced [3-5] $k_{C} / k_{L}$, where $k_{C}=C_{12} / \sqrt{C_{11} C_{22}}, k_{L}=L_{12} / \sqrt{L_{11} L_{22}}$, $C_{11}, C_{12}$, - elements of the per unit length capacity matrix, $L_{11}, L_{12}, L_{22}$ - elements of the per unit length inductances matrix of coupled lines. If $k_{C}=k_{L}$, the phase velocity of the even $v_{e}$ and odd $v_{o}$ modes in coupled lines are equal. Otherwise, inequalities $v_{e}>v_{o}$ or $v_{o}>v_{e}$ may be occurred.

In publications [2-6], matrix parameters were obtained for segments of equal and nonequal coupled strip lines. This made it possible to analyze the frequency characteristics of various devices taking into account the inhomogeneity of the dielectric filling of the cross section of the coupled lines. In [6], an analysis of quasi-T waves in a CSL with the ratio $k_{C} / k_{L}>3$ was carried out.

CSL are widely used in the design of various microwave devices. One of the most common types of devices based on CSL are directional couplers (DC) [7-9]. The wave properties of DC on coupled lines depend on the degree of electromagnetic coupling imbalance between the lines, i.e. from ratio $k_{C} / k_{L}$. It is considered that the best characteristics of directional couplers can be obtained if the coupling coefficient for the magnetic field is equal to the coupling coefficient for the electric field [8] when $k_{C}=k_{L}$. This applies only to traditional directional couplers on coupled lines with synchronous even and odd modes $[8,9]$. In the case of a significant difference in wave velocities "e" and "o" type in CSL, frequency characteristics of directional couplers have significant changes. Studying the relationship of DC parameters with respect to the ratio $k_{C} / k_{L}$ led to the creation of transdirectional couplers 
[10], in which the phase velocity of the even mode is 3 times higher than the phase velocity of the odd mode.

The first transdirectional coupler (TDC) on coupled lines was proposed in 2015 [11-14]. The coupler is implemented on a structure with a vertical substrate with high dielectric constant, which allows providing a three times ratio of normal wave velocities and acceptable impedance matching. Subsequent publications described a computer model of such a structure based on the method of numerical conformal transformations [15]. In these works, TDCs were investigated in the frequency domain.

In the present work, we consider the propagation of an ultrawideband pulse of the picosecond range. A pulse was applied to one of the TDC ports. An experimental measurement of pulsed signals at the output of three other ports and the reflected signal from the input port of the device was carried out. The spectra of the obtained impulse responses were calculated and the physical characteristics of the transdirectional coupler response under pulsed exposure were analyzed.

\section{DEVICE AND MEASUREMENT DIAGRAM}

The transdirectional coupler design in cross section is shown in Fig. 1 [10]. Coupled lines with width $W=2 \mathrm{~mm}$ are made on a substrate placed vertically to the grounded base. The gap between the bottom end of the substrate and the base was $D=0.5 \mathrm{~mm}$. The relative dielectric constant of the substrate was $\varepsilon_{r}=16.0$. Substrate material was Flan-16. The remaining dimensions of the cross section of the CSL: $\mathrm{H} 1=1.0 \mathrm{~mm} ; \mathrm{H} 2=12.5 \mathrm{~mm} ; \mathrm{H} 3=20.5 \mathrm{~mm}$. The design had coaxial-strip connectors. The appearance of the TDC design is shown in Fig. 2. The length of a line of coupled lines is $l=0.1 \mathrm{~m}$. The scheme of the experimental layout is shown in Fig. 3. The photograph of the experimental layout used to measure the impulse characteristics of the transdirectional coupler is shown in Fig. 4.

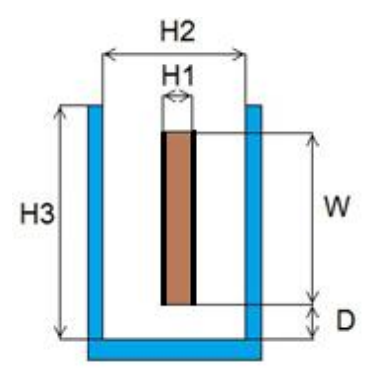

Fig. 1. Cross section of coupled strip lines of a transdirectional coupler 


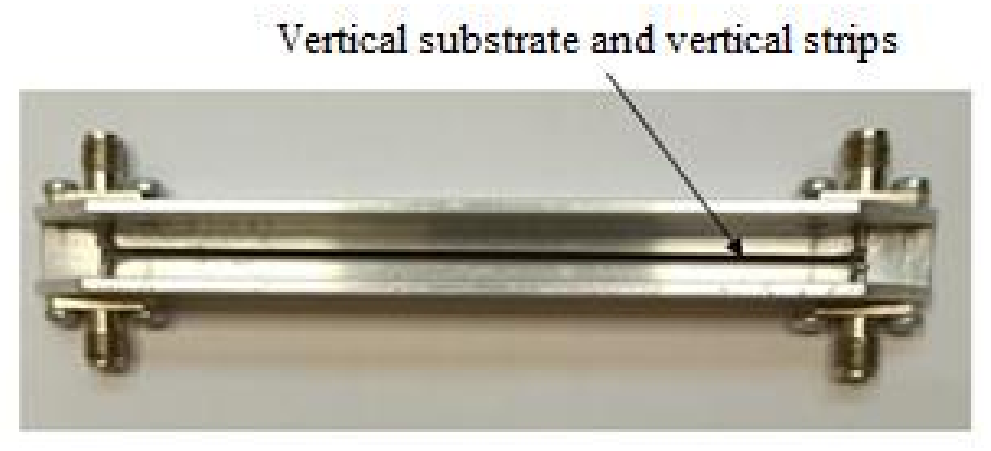

Fig. 2. Appearance of the transdirectional coupler

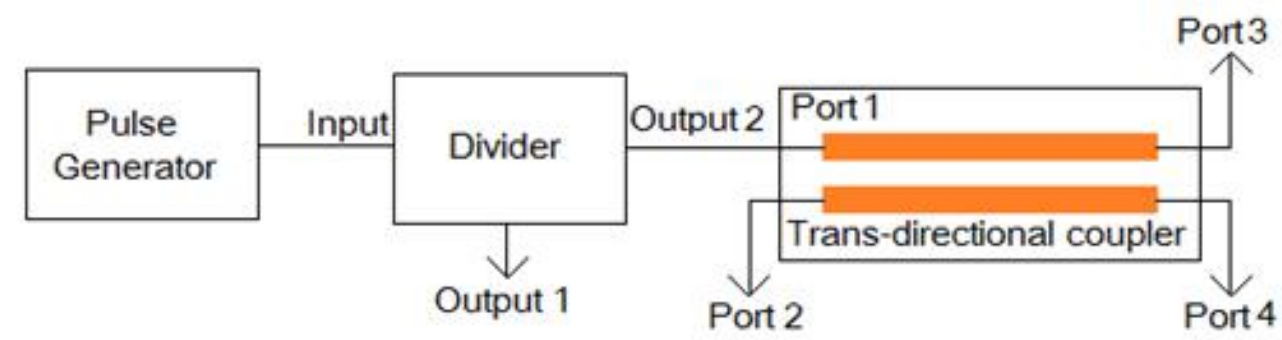

Fig. 3. Scheme of experimental layout used to study the impulse characteristics of a transdirectional coupler

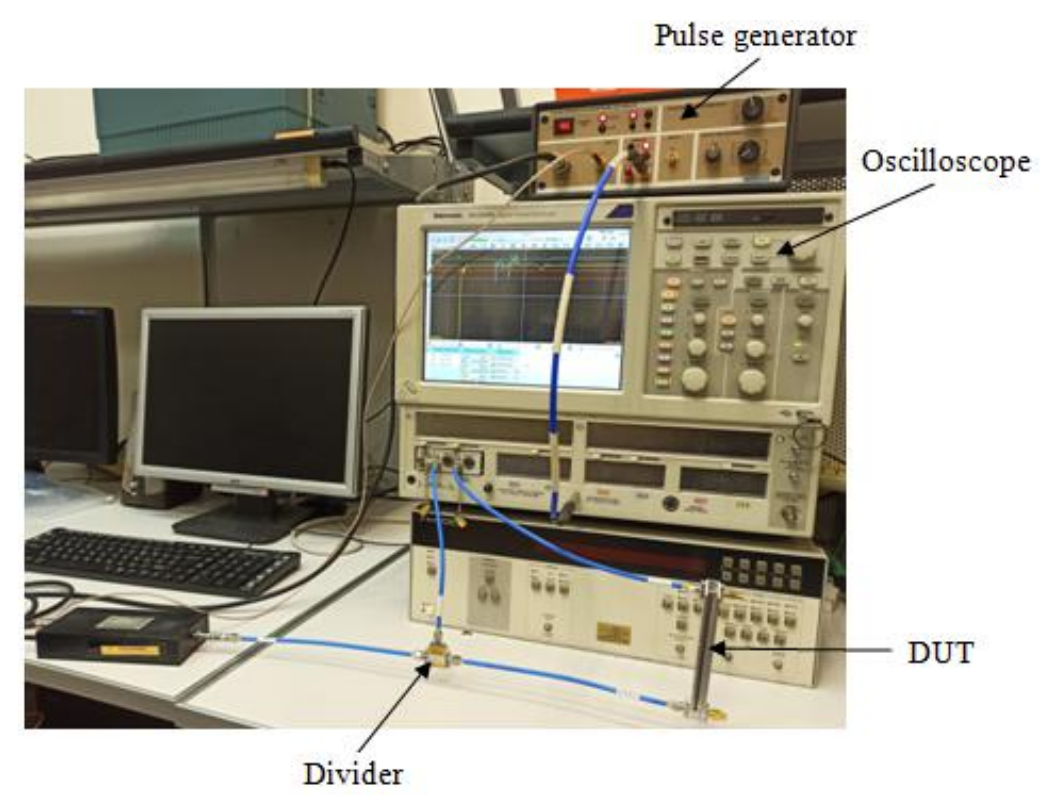

Fig. 4. Experimental layout used to measure the impulse characteristics of a transdirectional coupler

The experimental layout consists of: a pulsed generator including the reference generator Geozondas GZ1105DLP2 and the pulse shaper GZ1117DN-35; a splitter Picosecond 5372; oscilloscope DSA 8300; and a transdirectional coupler. The pulse generator is connected to the input of the divider, which has a decoupling of $14 \mathrm{~dB}$ with output 1 . The attenuation of the 
pulse received at the output 2 of the divider is $2 \mathrm{~dB}$. This pulse is fed through the coaxial cable to port 1 of the TDC. The DSA 8300 type oscilloscope has two inputs, which allows you to observe two pulse signals from output 2 of the divider and from one of the three ports 2 , 3, 4 of TDC. The oscilloscope is synchronized from the pulse generator. At the output 1 from the divider we observe a test pulse and reflected pulses from TDC. At the second input of the oscilloscope, we obtain pulses from other ports with loading unconnected ports by loads of 50 Ohms or without load (open). As a result, we observe complete information about the propagation of the test pulse in the device under study.

\section{EXPERIMENTAL RESULTS}

In the process of experimental studies, pulse signals were measured with different loads of ports 2,3 , and 4 . The task was to identify the characteristics of the propagation of the input pulse along the coupled lines of the device. Denote the load on the ports in accordance with the port numbers: $Z_{L O A D 1}, \ldots, Z_{L O A D 4}$. The loads of all ports in the experiment below were $Z_{L O A D 1}, \ldots, Z_{L O A D 4}=50$ Ohm. Fig. 5 illustrates the recording of a pulse at the output 2 of a divider with a duration of $40 \mathrm{ps}$ and an amplitude of $-0.7 \mathrm{~V}$. This pulse is fed to port 1 of the coupler. The time dependence of the voltage at the output of port 3 shows that the input pulse, when transmitted to port 3, was split into two pulses with amplitudes of $-0.26 \mathrm{~V}$ and $-0.22 \mathrm{~V}$. These pulses are delayed relative to the input pulse by $\tau_{31}=300 \mathrm{ps}$ and $\tau_{32}=600 \mathrm{ps}$, respectively.

Pulse signals on ports 2 and 4 are shown in Fig. 6. In this case, there is a more difficult picture of the input pulse transmit with the form of a sequence of pulses with a shape close to the shape of the input pulse and the ejection of pulses of a more complex shape and with a longer delay. The amplitude of the first pulse with a lower delay passed to port 2 is $-0.30 \mathrm{~V}$, and the first pulse received to port 4 is $-0.27 \mathrm{~V}$. 


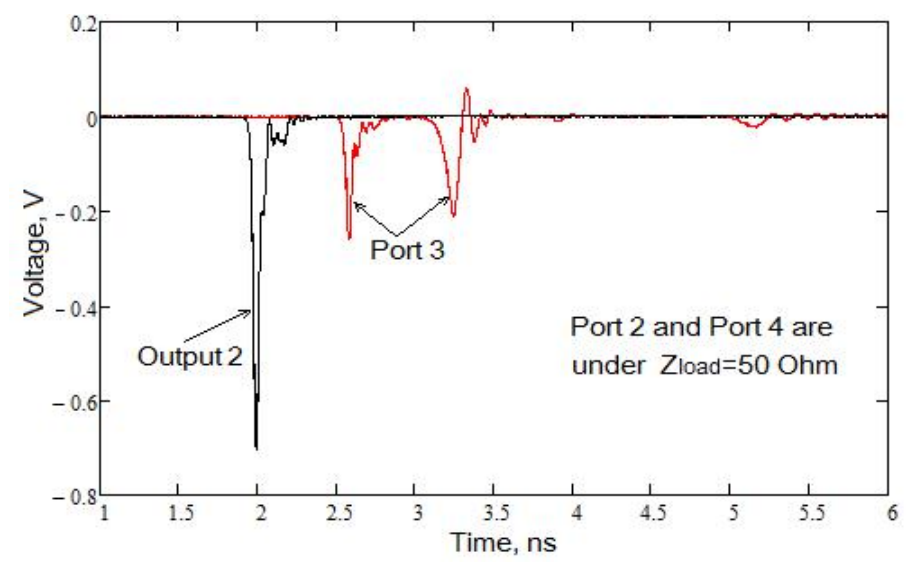

Fig. 5. Pulse signals at the input of the coupler (port1) and at the output (port 3) with loaded ports 2 and 4 at $50 \mathrm{Ohms}$

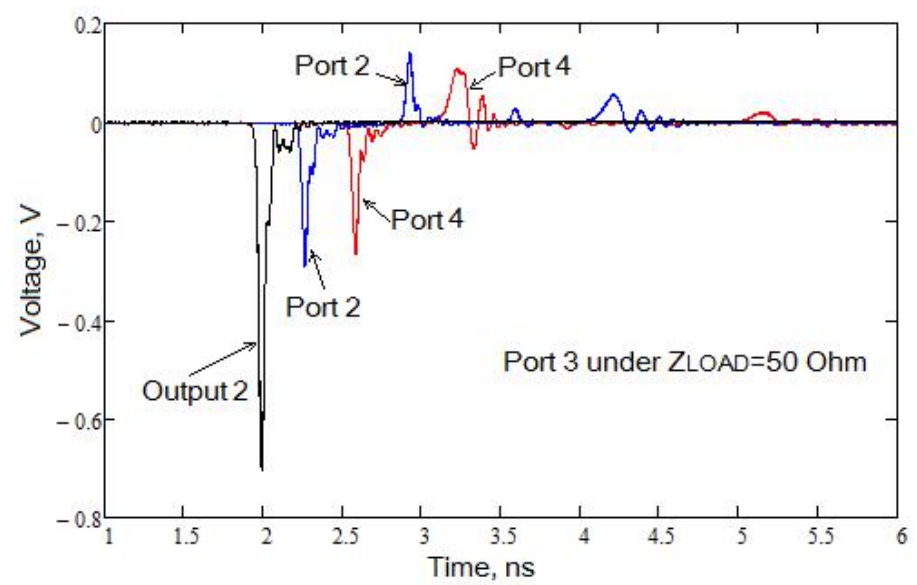

Fig. 6. Pulse signals at the coupler input (port1) and outputs (port 2 and port 4) with port 3 loading at 50 Ohm

To analyze the propagation mechanism of the input pulse along the connected lines of the transdirectional coupler, an important characteristic is the reflection of the input pulse from port 1 . Therefore, measurements of the reflected pulse are made. For this, a piece of coaxial cable was included between the divider and the tested device (see Fig. 4). As a result, the input pulse and the reflected pulse were separated by the time they arrived at the input of the oscilloscope. Figure 7 illustrates a comparison of input and reflected pulses. The maximum amplitude of the reflected pulse is $0.1 \mathrm{~V}$. This allows a qualitative assessment of the reflection coefficient modulus of the transdirectional coupler when exposed to an ultra-wideband pulse as a ratio of amplitudes $|\Gamma|=0,1 / 0,7=0,14$. 


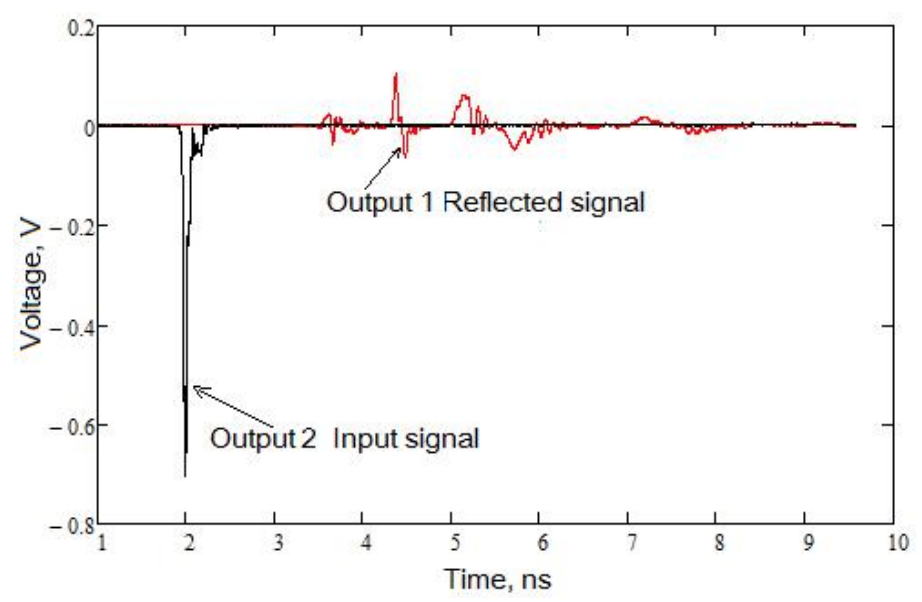

Fig. 7. Comparison of input and reflected pulses

It is known [10] that the frequency dependence of the input reflection coefficient in a wide frequency range is characterized by periodic poles. Therefore, during the studying TDC on the impact of a pulse signal, a significant reflected pulse was expected. But the received reflected signal shown in fig. 7, did not confirm our assumption. To explain the detected effect, an analysis was made of the spectra of the pulsed signal at the input of the transdirectional coupler and at ports 2, 3, and 4 (see Fig. 5, 6, and 7). Fig. 8 shows the envelopes of the spectra of the pulsed signals at the input of the transdirectional coupler, at its port 3 and the reflected signal from the input of the device under study.

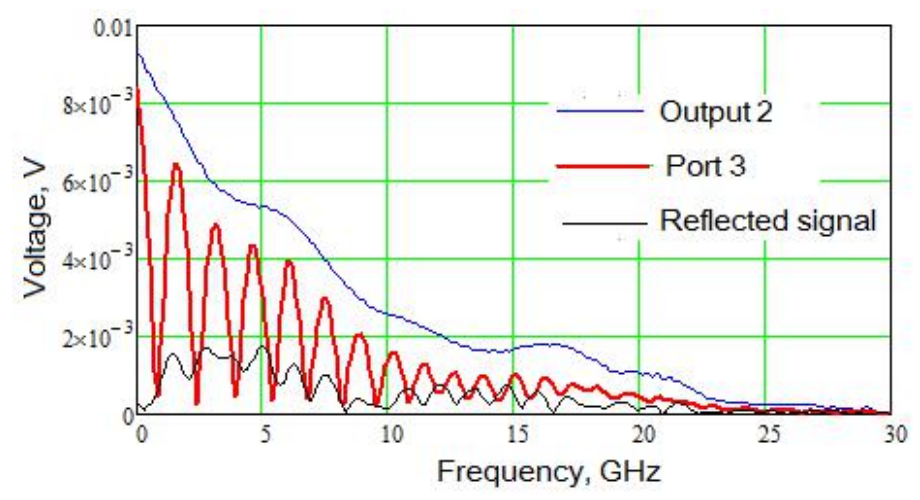

Fig. 8. Envelopes of the spectra of pulsed signals at output 2 (TDC input), at port 3 and reflected from TDC input (port 1)

The figure shows that the signal at output 2 has a continuous spectrum, the signal at port 3 has a trellised spectrum. The presence of relatively small reflections of the harmonic components from the input (shown in black), as well as losses in the coupled lines, reduces the transmission coefficient of the harmonics transmitted to port 3 from 0.85 to 0.70 in the frequency range from $0.1 \mathrm{GHz}$ to $8 \mathrm{GHz}$. In this case, the maximum reflection coefficient $|\Gamma|$ 
$=0.27$ is observed in the indicated frequency range of the transmitted harmonics at a frequency of $4.6 \mathrm{GHz}$.

Due to the presence of a lattice transmission spectrum and the absence of poles for the total reflection of the harmonic components of the ultra-wideband signal, the question arises of the direction of transmission of harmonics that have not passed to port 3 to other ports. The envelopes of the spectra of the signals at ports 2 and 4 were calculated (Fig. 9).

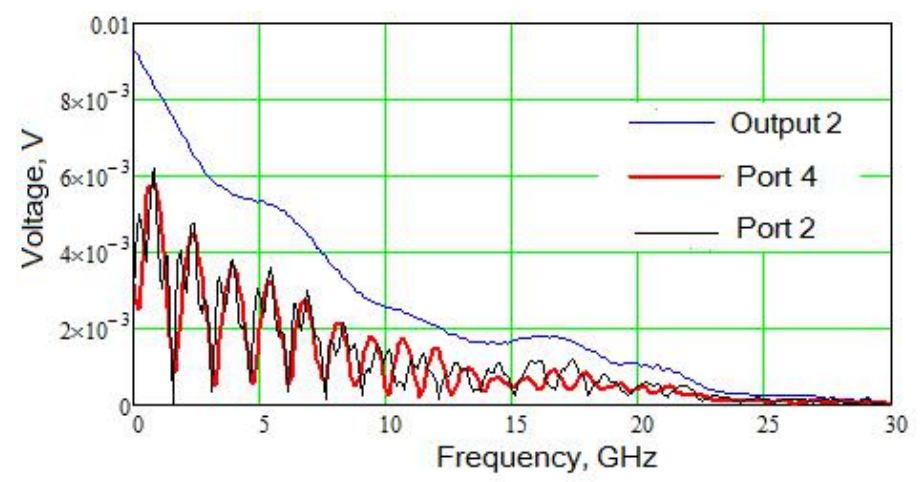

Fig. 9. Envelopes of the spectra of pulsed signals at output 2 (at the TDC input) and ports 2 and 4

Analysis of the spectral characteristics of Fig. 9 shows that harmonic components that did not pass through to port 3, with almost the same amplitudes up to a frequency of $8 \mathrm{GHz}$, fell into ports 2 and 4 .

\section{THE DISCUSSION OF THE RESULTS}

The measurements showed the propagation of an ultra-wideband pulse in coupled strip structures with strong unbalanced electromagnetic coupling between the lines. A $3 \mathrm{~dB}$ transdirectional coupler is used as measurement object. The experiment showed that a short pulse with a continuous spectrum up to $30 \mathrm{GHz}$ applied to port 1 is reflected from it with a relatively small (less than 0.27 ) reflection coefficient. In this case, the power of the harmonic components of the pulse signal is divided between ports 3,2 and 4. Harmonic components that have not passed to port 3 fall with approximately the same amplitudes into ports 2 and 4 , which are the beginning and end of the second strip conductor. An analysis of the phase relationships of the harmonic components in ports 2 and 4, which are down with minimal transient attenuation, shows a phase difference of 90 degrees up to a frequency of harmonic components of $8.23 \mathrm{GHz}$. The experimental features of the propagation of a short pulse in coupled TDC lines consist of combining the properties of directional power division and simultaneously directional filtering of the harmonic components of the signal over three ports. 
In other words, the property of the coupler is provided not only in the frequency domain, but also in the time domain.

\section{CONCLUSION}

Thus, the possibility of the propagation of ultra-wideband picosecond pulses without a significant loss of energy due to reflection from the entrance to transdirectional couplers based on coupled stripe lines is shown. However, in this case, the spectral components of the pulse are separated between the ports. The combination of directional division property and frequency selection (filtering) can be used in the formation of complex pulsed signals with spectral components on ports with equal amplitudes and orthogonal in phase. The results obtained in this article complement the understanding of the effect of splitting short pulses in modal filters based on coupled multi-wire lines [16].

\section{Acknowledgments}

This work was financially supported by the Ministry of Science and Higher Education of the Russian Federation, project No. FEWM-2020-0039 dated 01.03.2020. The measurements were carried out on the equipment of the Center of collective using of equipment "Impulse", project No. 075-15-2019-1644 of 11/08/2019, ID RFMEFI62119X0029.

\section{References}

[1] Vlostovskiy E. Theory of coupled transmission lines: Telecommun. and Radio Engrg., 1967, 21, pp. 87-93.

[2] Zysman, G.I., Johnson, A.K. Coupled Transmission Line Networks in an Inhomogeneous Dielectric Medium. IEEE Transactions on Microwave Theory and Techniques, 1969. Vol. 17. No 10, p. 753-759. DOI: 10.1109/TMTT.1969.1127055.

[3] Allen, J.L. Non-symmetrical coupled lines in an inhomogeneous dielectric medium. International Journal of Electronics. 1975. Vol. 38. No 3. P. 337-347. DOI: 10.1080/00207217508920406.

[4] Tripathi VK. Asymmetric coupled transmission lines in an inhomogeneous medium. IEEE Trans. Microwave Theory Tech. 1975, 23(9), p. 734-739. DOI: 10.1109/TMTT.1975.1128665. 
[5] Malyutin, N.D. Matrix parameters of non-identical, coupled striplines with a nonhomogeneous dielectric. Radio Eng. Electron Phys Vol. 21, Issue 12, Dec., Pp. 14-19.

[6] Vorob'ev P., Malyutin N., et al. Quasi-T-waves in devices using coupled strip lines with unbalanced electromagnetic coupling. Radio Engineering and Electronic Physics (English translation of Radiotekhnika i Elektronika). 1982. 27 (9). P. 38-44.

[7] Cristal E. G. Coupled-transmission-line directional couplers with coupled lines of unequal characteristic impedances. IEEE Microwave Theory and Technique, vol. MTT14, no. 7, pp. 337-346, Jul. 1966. DOI: 10.1109/TMTT.1966.1126266.

[8] Cohn S. B. and Levy R. History of microwave passive components with particular attention to directional couplers. IEEE Trans. Microwave Theory and Technique, vol. MTT-32, no. 9, pp. 1046-1054, Sep. 1984. DOI: 10.1109/TMTT.1984.1132816.

[9] Matthaei G, Young L, Jones EM T. Microwave filters, impedance matching networks and coupling structures. Norwood (MA): Artech House; 1985. DOI: 10.1109/PROC.1965.4048.

[10] Sychev A.N., Struchkov S.M., Rudyi N.Y. Synthesis of an ideal phase ratio for a coupled-line coupler with given type of directivity, Proc. TUSUR, 2017, vol. 20, no. 2, pp. 15-18. (In Russ.).

[11] Pat. 2585884 RU Transdirectional coupled line coupler with vertical plate / A.N.Sychev, S.M.Struchkov, et al. TUSUR, Appl. № 2015108565/28, Filed: 11.03.2015, Publ.: 10.06.2016, Bul. № 16 .

[12] Sychev A.N., Struchkov S.M., et al. A novel transdirectional coupler based on vertically installed planar circuit. Proc. of the 45-th Eur. Microw. Conf., Sept. 2015, Paris, France, pp. 283-286. DOI: 10.1109/EuMC.2015.7345755.

[13] Sychev A.N., Struchkov S.M., Rudyi N.Y. A transdirectional coupled-line coupler with a vertical insert. Proc. 25th Int. Crimean Conf. "Microwave \& Telecommunication Technology" (CriMiCo'2015), Sept. 2015, Sevastopol, Russia, pp. 547-549.

[14] Sychev A.N., Struchkov S.M., Rudyi N.Y. Three-section transdirectional coupler of Xband. Proc. of the TUSUR, 2015, no. 4 (36). - pp. 12-16 (in Russ.)

[15] Sychev A.N., Struchkov S.M., Rudyi N.Y., Salnikov A.S. Modeling of the vertically installed planar coupled lines by the numerical conformal transformation technique. IEEE MTT-S Int. Conf. on Numerical Electromagnetic and Multiphysics Modeling and Optimization (NEMO), May 2017, Sevilla, Spain.- P.124-126. DOI: 10.1109/NEMO.2017.7964202. 
[16] Belousov, A.O., Gazizov T.R. Simulation of the time response in multiconductor microstrip modal filters with separate accounting for losses in conductors and dielectrics. Moscow Workshop on Electronic and Networking Technologies, MWENT 2018 ProceedingsVolume 2018-March, 12 April 2018, Pp. 1-5. DOI: 10.1109/MWENT.2018.8337216. 